\title{
Validity and Limitations of the Median-Line Integral Equation Technique in the Scattering by Material Strips of Sub-Wavelength Thickness
}

\author{
Ilya O. Sukharevsky, Member, IEEE, Olga V. Shapoval, Student Member, IEEE, \\ Ayhan Altintas, Senior Member, IEEE, and Alexander I. Nosich, Fellow, IEEE
}

\begin{abstract}
Considered is the 2-D scattering of a plane wave by a thin flat material strip. The data obtained by using the empirical method of generalized boundary conditions and singular integral equations on the strip median line are compared with the results of solving the Muller boundary integral equation that takes full account of strip thickness. Discretization of integral equations in both cases is performed using the Nystrom methods that lead to convergent algorithms. Numerical results cover $E$ and $H$ polarizations and two types of thin strips: conventional dielectric and metal in the optical range. The validity and limitations of approximate model are established and discussed.
\end{abstract}

Index Terms-Generalized boundary conditions, integral equations, scattering, thin strip.

\section{INTRODUCTION}

$\mathbf{E}$ FFICIENT numerical method for the analysis of 2-D wave scattering by a thin material strip (see Fig. 1), based on generalized boundary conditions (GBC) and corresponding singular integral equations (SIE) on the strip median line, was suggested in [1]. In fact, these SIE are a 2-D version of similar equations derived earlier in [2], [3]. The "added value" of [1] is solution of SIE with a meshless Nystrom-type discretization that provides both guaranteed convergence and very small size of a matrix to be inverted. This has allowed, apparently for the first time, making fast and accurate computations of the scattering by finite gratings of hundreds of strips [4], i.e., by scatterers having total size in hundreds of wavelengths. However, since the considered SIEs are obtained using the GBC derived for an infinite thin material slab, their applicability in certain cases (e.g., shorter and thicker finite strips, resonances,

Manuscript received November 25, 2013; revised February 11, 2014; accepted April 02, 2014. Date of publication April 08, 2014; date of current version July 02,2014 . This work was supported in part by the National Academy of Sciences of Ukraine via the State Target Program "Nanotechnologies and Nanomaterials."

I. O. Sukharevsky and A. Altintas are with the Communication and Spectrum Management Research Center (ISYAM) and Electrical and Electronics Engineering Department, Bilkent University, 06800 Bilkent, Ankara, Turkey (e-mail: i.sukharevsky@gmail.com).

O. V. Shapoval and A. I. Nosich are with the Laboratory of Micro and Nano Optics, Institute of Radio-Physics and Electronics, National Academy of Sciences of Ukraine, Kharkiv 61085, Ukraine (e-mail: olga.v.shapoval@gmail. com).

Color versions of one or more of the figures in this paper are available online at http://ieeexplore.iee.org.

Digital Object Identifier 10.1109/TAP.2014.2316295

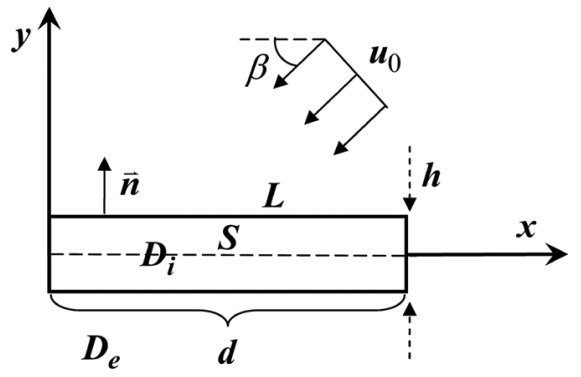

Fig. 1. Cross-section of a free-standing thin material strip of relative dielectric permittivity $\varepsilon$, width $d$ and the thickness $h$, with rounded edges (see text for details), illuminated by a plane wave incident under the angle $\beta$.

and grazing incidence) can be questioned and has to be a subject of research.

This is the goal of our paper. Validity and limitations of the GBC-SIE approach can be established by confronting its numerical results with the reference ones. As the reference numerical solution, one has to use sufficiently accurate data obtained with another numerical method, which 1) takes full account of strip thickness and 2) possesses, similarly to GBC-SIE, guaranteed convergence, i.e., allows one to control accuracy, to machine precision, by varying the matrix order.

In the scattering of waves by 2-D homogeneous dielectric bodies, boundary IE techniques are one of the most popular computational tools. Still amazingly many researchers use the forms of such IE that are not fully equivalent to the original boundary-value problem, being corrupted by the presence of infinite set of spurious real-valued eigenvalues [5], [6]. As discussed in [7]-[9], this pitfall can be avoided by using the Muller boundary IE (MBIE) that is a pair of coupled second-kind IE for the field tangential components [10]. Unlike the combined-field IE, MBIE has no spurious eigenvalues (the former only pushes them off from the real axis). Additionally, smooth or integrable kernels make it a Fredholm second-kind IE, and therefore Fredholm theorems guarantee convergence of both local mesh-based and global Galerkin discretizations.

Still other numerical treatment of MBIE that guarantees convergence is the Nystrom-type meshless discretization using interpolation polynomials and quadrature formulas. Here, several schemes have been devised recently, most notably for contours allowing smooth $2 \pi$-periodic parameterization [8], [9]. In particular, the economic and robust algorithm of [8] exploits splitting all kernels of MBIE into logarithmic and smooth parts and 
uses two different quadrature formulas for integrating them explicitly. However this treatment must be modified if the contour is only piece-wise smooth.

In the remainder of this paper, we briefly present the MBIE and its numerical solution in Section II and the GBC-SIE and the Nystrom algorithm in Section III. Section IV contains comparative data on the scattering of $\mathrm{E}$ and $\mathrm{H}$-polarized plane waves by two types of thinner-than-wavelength strips: non-dispersive dielectric strips and dispersive metal ones in the optical range. Conclusions are summarized in Section V. Throughout the paper, time dependence $\sim \exp (-i \omega t)$ is assumed and omitted.

\section{Muller Boundary Integral Equation Method}

\section{A. Formulation and Muller Equations}

Consider an electromagnetic plane wave incident normally at the angle $\beta$ on a flat material nonmagnetic strip (infinite along the $z$-axis) as depicted in Fig. 1 . The strip $D_{i}=\{(x, y): 0 \leq$ $x \leq d,|y| \leq h / 2\}$ has complex relative permittivity or dielectric function $\varepsilon(\lambda)$, width $d$, thickness $h$, and cross-section contour $L$. The host medium is free space.

Assume that the incident plane-wave $z$-component of the E $(\mathrm{H})$ field in the case of the $\mathrm{E}(\mathrm{H})$ polarization is given as $U^{i n c}(\vec{r})=e^{-i k_{0}(x \cos \beta+y \sin \beta)}$, where $\vec{r}=(x, y), k_{0}=\omega / c$, and $c$ is the free-space light velocity. In the presence of strip, the total field $U(\vec{r})$ is a sum of this function and the scattered field, $U^{s c}(\vec{r})$. The latter function must satisfy relevant Helmholtz equation inside (in $D_{i}$ ) and outside the strip (in $D_{e}$ ) and the continuity of tangential components across $L$. For the uniqueness of the solution, $U^{s c}(\vec{r})$ must satisfy Sommerfeld radiation condition and condition of local energy finiteness.

Derivation of MBIE is based on the use of the Green's formulas. In the 2-D case, all details of such derivation can be found in [7], [8], so that we present only the resulting equations:

$$
\begin{aligned}
U(\vec{r}) & +\int_{L} K_{11}\left(\vec{r}, \vec{r}^{\prime}\right) U\left(\vec{r}^{\prime}\right) d s^{\prime}-\int_{L} K_{12}\left(\vec{r}, \vec{r}^{\prime}\right) V\left(\vec{r}^{\prime}\right) d s^{\prime} \\
= & U^{i n c}(\vec{r}) \\
\frac{1+p}{2} V(\vec{r}) & +\int_{L} K_{21}\left(\vec{r}, \vec{r}^{\prime}\right) U\left(\vec{r}^{\prime}\right) d s^{\prime}-\int_{L} K_{22}\left(\vec{r}, \vec{r}^{\prime}\right) V\left(\vec{r}^{\prime}\right) d s^{\prime} \\
= & V^{i n c}(\vec{r}), \quad \vec{r} \in L .
\end{aligned}
$$

Here, $V(\vec{r})=\partial U(\vec{r}) / \partial n$ is the limit value of the normal derivative of the total field on the closed contour $L$ of the scatterer from the inner side of it, the normal unit vector $\vec{n}$ is directed to the outer domain $D_{e}$ (free space), $d \vec{r}^{\prime}$ is the elementary arc length, and the constant is $p=1$ in the E-polarization case and $p=1 / \varepsilon$ in the H-polarization case.

The kernels of MBIE have the following form:

$$
\begin{aligned}
& K_{11}=\frac{\partial G_{i}}{\partial n^{\prime}}-\frac{\partial G_{e}}{\partial n^{\prime}}, \quad K_{12}=G_{i}-p G_{e}, \\
& K_{21}=\frac{\partial^{2} G_{i}}{\partial n \partial n^{\prime}}-\frac{\partial^{2} G_{e}}{\partial n \partial n^{\prime}}, \quad K_{22}=\frac{\partial G_{i}}{\partial n}-p \frac{\partial G_{e}}{\partial n}
\end{aligned}
$$

where $G_{(i, e)}=G_{(i, e)}\left(\vec{r}, \vec{r}^{\prime}\right)=(i / 4) H_{0}^{(1)}\left(k_{i, e} \rho\right)$ are the Green's functions of the corresponding homogeneous media, $k_{e}=k_{0}, k_{i}=k_{0} \sqrt{\varepsilon}$, and $\rho=\left|\vec{r}-\vec{r}^{\prime}\right|$.

\section{B. Discretization and Numerical Algorithm}

If $\rho=\left|\vec{r}-\vec{r}^{\prime}\right|$ is a Lyapunov curve, then the kernel functions have at most weak logarithmic singularities (see [7], [8]), and (1), (2) are Fredholm second kind IEs. This guarantees convergence of usual numerical schemes of its discretization. However, the assumption of $L$ to be a Lyapunov curve is essential here, since (1), (2) are derived by using theorems of classical potential theory [11], and in non-regular points of contour the unit normal vector and some field components and their derivatives are not defined. In order to overcome this problem, one can approximate irregular curve by a smooth one and then apply quadratures for the $2 \pi$-periodic functions [8]. For example, rectangular shape can be smoothed using the trigonometric polynomial approximation (truncated Fourier series) [12] or superellipse formula [7]. However Fourier series are known to diverge for the contours with edges and even with finite jumps of curvature. The superellipse curve is not well suited to our problem, because parameterization with the polar angle requires very dense mesh near to the ends of a thin strip with high ratio of $d / h$.

Therefore, as shown in Fig. 1, we subdivide the strip contour $L$ into separate smooth segments that is four straight intervals, extract logarithmic singularities from (3) and (4), and apply quadratures on each segment. The discretization performed in this manner is not sensitive to the irregularities of the contour so far as the interpolation nodes do not coincide with edge points. Full analysis of the kernels at $\vec{r} \rightarrow \vec{r}^{\prime}$ can be found in [7], [8]. Denoting the contour curvature $\varsigma(\vec{r})$, we introduce new continuous kernels as follows:

$$
\begin{aligned}
& \tilde{K}_{11} \\
& = \begin{cases}K_{11}=\frac{i}{4}\left[-k_{i} H_{1}^{(1)}\left(k_{i} \rho\right)+k_{e} H_{1}^{(1)}\left(k_{e} \rho\right)\right] \frac{\partial \rho}{\partial n^{\prime}}, & \vec{r} \neq \vec{r}^{\prime} \\
0, & \vec{r}=\vec{r}^{\prime}\end{cases}
\end{aligned}
$$

$\tilde{K}_{12}$

$$
= \begin{cases}K_{12}=\frac{i}{4}\left[H_{0}^{(1)}\left(k_{i} \rho\right)-p H_{0}^{(1)}\left(k_{e} \rho\right)\right], & \vec{r} \neq \vec{r}^{\prime} \\ (1-p)\left(\frac{i}{4}-\frac{C}{2 \pi}\right)-\frac{1}{2 \pi}\left(\ln \frac{k_{i}}{2}-p \ln \frac{k_{e}}{2}\right), & \vec{r}=\vec{r}^{\prime}\end{cases}
$$

$$
\begin{aligned}
& \tilde{K}_{22} \\
& = \begin{cases}K_{22}=\frac{i}{4}\left[p k_{e} H_{0}^{(1)}\left(k_{e} \rho\right)-k_{i} H_{0}^{(1)}\left(k_{i} \rho\right)\right] \frac{\partial \rho}{\partial n}, & \vec{r} \neq \vec{r}^{\prime} \\
\frac{1-p}{4 \pi} \varsigma(\vec{r}), & \vec{r}=\vec{r}^{\prime}\end{cases}
\end{aligned}
$$

$$
= \begin{cases}\tilde{K}_{21} & \\ & K_{21}=\frac{i}{8}\left[k_{e}^{2} H_{0}^{(1)}\left(k_{e} \rho\right)-k_{i}^{2} H_{0}^{(1)}\left(k_{i} \rho\right)\right. \\ & \left.k_{i}^{2} H_{2}^{(1)}\left(k_{i} \rho\right)-k_{e}^{2} H_{2}^{(1)}\left(k_{e} \rho\right)\right] \frac{\partial \rho}{\partial n} \frac{\partial \rho}{\partial n^{\prime}} \\ +\frac{i}{4}\left[k_{e}^{2} H_{1}^{(1)}\left(k_{e} \rho\right)-k_{i}^{2} H_{1}^{(1)}\left(k_{i} \rho\right)\right] \frac{\partial^{2} \rho}{\partial n \partial n^{\prime}}, & \vec{r} \neq \vec{r}^{\prime} . \\ \frac{k_{i}^{2}-k_{e}^{2}}{4 \pi}\left(\frac{i}{2}-C-\ln \frac{k_{i}}{2}\right) & \\ -\frac{1}{4 \pi}\left(k_{i}^{2} \ln \frac{k_{i}}{2}-k_{e}^{2} \ln \frac{k_{e}}{2}\right), & \vec{r}=\vec{r}^{\prime}\end{cases}
$$

Introduce $N$ subsections of the lengths $\Delta_{j}(j=1, \ldots, N)$ of the segments of $L$ and assume that unknown functions are constants at each subsection. Then, after applying the rectangle 
rule for numerical integration, we obtain a matrix equation with respect to the unknowns

$$
\begin{aligned}
& U\left(\vec{r}_{i}\right)+\sum_{j=1}^{N} \tilde{K}_{11}\left(\vec{r}_{i}, \vec{r}_{j}\right) U\left(\vec{r}_{j}\right)\left|\Delta_{j}\right| \\
&-\sum_{j=1}^{N} \tilde{K}_{12}\left(\vec{r}_{i}, \vec{r}_{j}\right) V\left(\vec{r}_{j}\right)\left|\Delta_{j}\right| \\
&-(p-1) V\left(\vec{r}_{i}\right) \int_{\Delta_{i}} \ln \rho d s^{\prime}=U^{i n c}\left(\vec{r}_{i}\right), \\
&\left(\frac{1}{2}\right)(1+p) V\left(\vec{r}_{i}\right)+\sum_{i=1}^{N} \tilde{K}_{21}\left(\vec{r}_{i}, \vec{r}_{j}\right) U\left(\vec{r}_{j}\right)\left|\Delta_{j}\right| \\
&-\sum_{i=1}^{N} \tilde{K}_{22}\left(\vec{r}_{i}, \vec{r}_{j}\right) V\left(\vec{r}_{j}\right)\left|\Delta_{j}\right| \\
&+\frac{k_{i}^{2}-k_{e}^{2}}{4 \pi} U\left(\vec{r}_{i}\right) \int_{\Delta_{i}} \ln \rho d s^{\prime}=V^{i n c}\left(\vec{r}_{i}\right) .
\end{aligned}
$$

As $\rho=\left|\vec{r}-\vec{r}^{\prime}\right|$, the value of $\int_{L_{i}} \ln \rho d s^{\prime}$ can be obtained analytically.

In computations, we have taken $N=600$ in the case of a half-wavelength dielectric strip with $\varepsilon=4$, and 200 to 1000 in the case of silver strip of $d=150$ to $900 \mathrm{~nm}$. This guaranteed the accuracy, in the far field, of the order of $10^{-4}$ and $10^{-3}$, respectively (see Appendix II). Larger $N$ for a metal strip are explained by the larger values of $|\varepsilon|$.

\section{Generalized Boundary Conditions-Singular INTEGRAL EQUATION METHOD}

\section{A. Dielectric Strip, E-Polarization}

GBC-SIE is an empirical method, in the sense that it does not follow directly from MBIE in fully justified manner; instead it uses assumptions valid for thin infinite layers.

In brief, these assumptions are as follows: if $h \ll \lambda\left(k_{0} h \ll\right.$ $1)$, one can shrink the strip cross-section $D_{i}$ to its median line, $S=\{(x, 0): x \in[0, d], y=0\}$, exclude the field inside the strip from consideration, and impose, at $S$, two-side GBC first derived in [13] for an infinite thin material slab (see Appendix I). We will use the following form of GBC adopted in [3] as more transparent:

$$
\begin{aligned}
\left(\frac{1}{2}\right) & {\left[\vec{E}_{T}^{+}(x, 0)+\vec{E}_{T}^{-}(x, 0)\right] } \\
= & R Z_{0} \vec{n} \times\left[\vec{H}_{T}^{+}(x, 0)-\vec{H}_{T}^{-}(x, 0)\right] \\
\left(\frac{1}{2}\right) & {\left[\vec{H}_{T}^{+}(x, 0)+\vec{H}_{T}^{-}(x, 0)\right] } \\
= & -Q Z_{0}^{-1} \vec{n} \times\left[\vec{E}_{T}^{+}(x, 0)-\vec{E}_{T}^{-}(x, 0)\right] .
\end{aligned}
$$

Here, $\vec{n}=(0,1)$ is the unit normal vector, $Z_{0}$ is the free-space impedance, the indices \pm correspond to the field limit values at the top and bottom sides of the strip, respectively. These GBC have been derived for an infinite thin slab illuminated by a plane wave [13]-[16]. The coefficients $R$ and $Q$ are called the electric and magnetic resistivities, which have several forms depending on the slab $\varepsilon$. If the contrast is high, $|\varepsilon| \gg 1$, then [13], [14], [16], [3]

$$
R=i\left(\frac{\nu^{-1}}{2}\right) \cot \left(\frac{k_{0} h \nu}{2}\right), \quad Q=i\left(\frac{\nu}{2}\right) \cot \left(\frac{k_{0} h \nu}{2}\right)
$$

where $\nu(\lambda)=\sqrt{\varepsilon(\lambda)}$ is the refractive index. If, alternatively, the contrast is low, $k_{0} h|\varepsilon-1| \ll 1$, then [15]

$$
R=i\left[\nu(\varepsilon-1) k_{0} h\right]^{-1}, \quad Q=i \nu\left[(\varepsilon-1) k_{0} h\right]^{-1} .
$$

The GBC (11) is approximate, however it is clearly a big step ahead from the PEC condition, to which it turns if $R=0$ and $Q=\infty$ (see Appendix I). In [17], a modified version of (12) has been proposed that keeps validity for $\varepsilon \rightarrow 1$ by taking into account the compensation for the reduction of the slab area to the line of zero thickness. According to [17],

$$
R^{*}=\frac{\vartheta-R-\vartheta^{2} R}{4 \vartheta R-\vartheta^{2}-1}, \quad Q^{*}=\frac{\vartheta-Q-\vartheta^{2} Q}{4 \vartheta Q-\vartheta^{2}-1}
$$

where $\vartheta=i \cot \left(k_{0} h / 4\right)$, and $R$ and $Q$ are taken from (12).

Note that GBC (11) has the meaning of Ohm's law for the effective electric and magnetic currents, which correspond to the difference terms in their right-hand parts. Other form of effective BC involving field derivatives has been discussed in [15], [18]. We will not present here any results computed with such modified GBC. This is because they coincide with the data based on (11)-(12) within $1 \%$ for all strips considered in our paper. If the strip median is not straight then GBC takes more complicated form involving cross-resistivities [3], [18].

When GBC (11) is imposed at a finite strip, the uniqueness of solution is again provided by the condition of local finiteness of power and the radiation condition. Note that the field singularities at the resistive strip endpoints are different from the right-angle dielectric wedge as revealed in [19].

Although GBC (11) does not depend on the width $d$, it is natural to expect that for very narrow strips, say $d<\lambda$, its use is too far from infinite-slab situation. Still note that GBC (11) and (12) have already been used in the analysis of infinite flat gratings of dielectric and metal strips [20], [21]. Their partial validation was done in [20] using the volume IE in the E-polarization case for $d<\lambda$.

\section{B. Singular Integral Equations and Numerical Algorithm}

We seek the scattered field as a sum of single-layer and double-layer potentials

$$
U^{s c}(\vec{r})=k_{0} \int_{S} v\left(\vec{r}^{\prime}\right) G\left(\vec{r}, \vec{r}^{\prime}\right) d s^{\prime}+\int_{S} w\left(\vec{r}^{\prime}\right) \frac{\partial G\left(\vec{r}, \vec{r}^{\prime}\right)}{\partial \vec{r}^{\prime}} d s^{\prime}
$$

where $U(\vec{r})=U^{s c}(\vec{r})+e^{-i k_{0}(x \cos \beta+y \sin \beta)}$ is either $H_{z}(\vec{r})$ or $E_{z}(\vec{r})$ depending on the polarization, and $G\left(\vec{r}, \vec{r}^{\prime}\right)$ is the free-space Green's function. From the properties of the limit values of potentials it follows that unknown functions are $v(\vec{r})=\partial / \partial \vec{n}(\vec{r})\left[U^{+}(\vec{r})-U^{-}(\vec{r})\right]$ and $w(\vec{r})=U^{+}(\vec{r})-U^{-}(\vec{r})$.

Using (15) in GBC (11) yields two independent IEs of the second kind. In the H-polarization case, after the introduction of the strip parameterization as $x=x(t)=d(t+1) / 2, y=$ 
$y(t) \equiv 0$ for $t \in[-1,1]$ and new smooth unknown function $\tilde{w}(t)=w(t)\left(1-t^{2}\right)^{-1 / 2}$, we obtain

$$
\begin{gathered}
4 Q v\left(t_{0}\right)+\kappa \int_{-1}^{1} v(t) H_{0}^{(1)}\left(\kappa\left|t-t_{0}\right|\right) d t=f_{v}\left(\kappa, t_{0}\right) \\
4 R \tilde{w}\left(t_{0}\right) \sqrt{1-t_{0}^{2}} \\
+\int_{-1}^{1} \tilde{w}(t) \sqrt{1-t^{2}} \frac{H_{1}^{(1)}\left(\kappa\left|t-t_{0}\right|\right)}{\left|t-t_{0}\right|} d t=f_{w}\left(\kappa, t_{0}\right) .
\end{gathered}
$$

In the E-polarization case, the quantities $R$ and $Q$ exchange places. Here, $\kappa=k_{0} d / 2$ is the normalized frequency and the terms $f_{v}(\kappa, t)=4 i e^{-i \kappa(t+1) \cos \beta}$ and $f_{w}(\kappa, t)=4 \sin \beta e^{-i \kappa(t+1) \cos \beta}$ are defined by the incident wave. Note that SIE (16) has logarithmic singularity and (17) is a hyper-singular SIE whose integral operator should be understood in the sense of Hadamard's finite part.

Further we extract the singularities and discretize each SIE using a Nystrom-type method approximating the unknowns by polynomials and using quadrature rules of interpolation type [1], [4]. For (16), we use the Gauss-Legendre quadrature formulas of the $N$ th order with nodes in the nulls of Legendre polynomials. For (17), we use weighted Gauss-Chebyshev quadrature formulas of the $N$ th order with nodes in the nulls of Chebyshev polynomials of the second kind. This yields two independent matrix equations for the surface currents. The theorems on quadratures ensure quick convergence of their solutions to the accurate ones if $N \rightarrow \infty$ (at least as $1 / N$ ), see Appendix II. All data presented below have been computed with $10^{-4}$ accuracy $(N=50)$. Note that unlike PEC-strip SIE [22], (16), (17) cannot provide exponential convergence.

\section{Numerical Results}

To study validity and limitations of the GBC-SIE approach, we have compared its results with those of MBIE. We have considered the $\mathrm{E}$ and $\mathrm{H}$-polarized plane wave incident on non-dispersive dielectric strips and dispersive metal strips, with varying strip width, thickness, permittivity, and incidence angle. The total scattering cross-section and the radar cross-section (TSCS and RCS) have been computed.

\section{A. Dielectric Strip, E-Polarization}

First of all, we compare the above mentioned methods and models with Galerkin's method of Richmond applied to the volume IE [23] for the edge-on E-wave scattering by a thin ( $h=0.025 \lambda$ ) dielectric lossy strip with $\varepsilon=4+0.4 i$. In Fig. 2, we demonstrate the corresponding plots of the normalized RCS versus the incidence angle; the inset explains the origin of results. Note that both MBIE and GBC-SIE algorithms were adapted to keep four-digit accuracy.

As one can see, for such a small thickness and moderate $\varepsilon$, it is sufficient to apply GBC with either the low-contrast $R$ and $Q$ (13) or the compensated high-contrast ones (14). This model is accurate at any angle of incidence while the non-compensated high-contrast model (12) fails at the grazing. In general, the edge-on incidence $(\beta=0)$ is the least favorable for using the GBC. However, even in this case both low-contrast (13) and

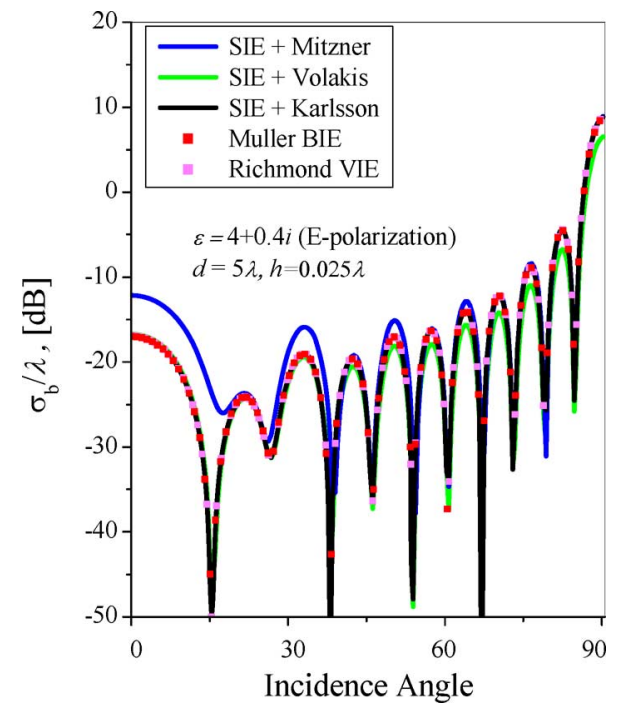

Fig. 2. Normalized RCS as a function of the incidence angle of the E-wave for the scattering by strip with $d=5 \lambda, h=0.025 \lambda$ and $\varepsilon=4.0+0.4 i$.

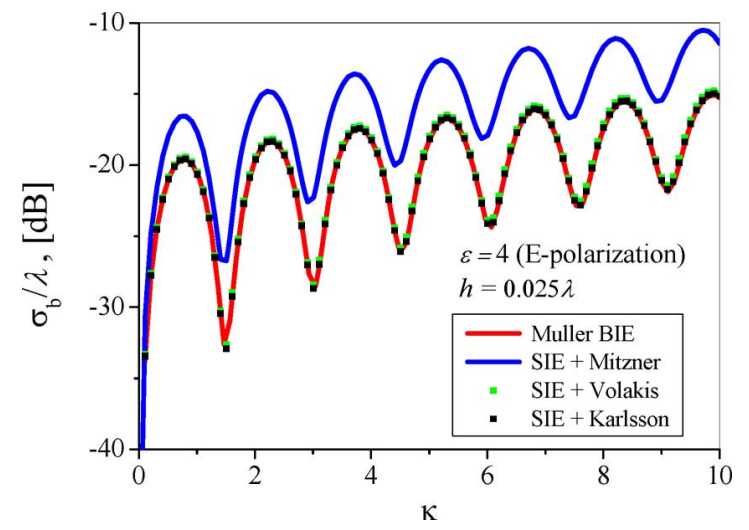

Fig. 3. Normalized RCS versus the normalized frequency $\kappa$ for the edge-on scattering of E-wave $(\beta=0)$ by a dielectric strip of $h=0.025 \lambda$ with $\varepsilon=4.0$.

compensated high-contrast (14) models yield accurate dependences of RCS in the wide range of frequencies (Fig. 3). Deep periodic minima in RCS are explained by the antiphase interference of reflections from the leading and trailing edges of the $n \lambda / 2$-wide strip, where $n=1,2, \ldots$

Further we have computed the scattering by strips made of the dielectrics with relatively large values of Re $\varepsilon$. In Fig. 4, we compare spectra of TSCS for a lossless strip with $\varepsilon=20$ and thickness $h / d=0.01$ under the broadside illumination $(\beta=$ $\pi / 2)$. As visible, the compensated model of (14) is the best, although low-contrast model of (13) can be also used with a $2 \%$ or better accuracy if $\kappa<2.5$, i.e., where $k_{0} h|\varepsilon-1|<0.5$. All models reproduce almost periodic Fabry-Perot resonances on the principal E-polarized surface wave of the corresponding slab. Still the largest errors are near these resonances because of the shifts of resonance frequencies.

The relative errors versus varying frequency are shown in Fig. 5 where MBIE results are taken as a reference, so that

$$
\text { error }=\frac{\left|\sigma_{s c}^{G B C-S I E}-\sigma_{s c}^{M B I E}\right|}{\sigma_{s c}^{M B I E}} .
$$

One can see that the error in Karlsson's model does not exceed $2 \%$ in contrast to $12 \%$ for the model of (12). 


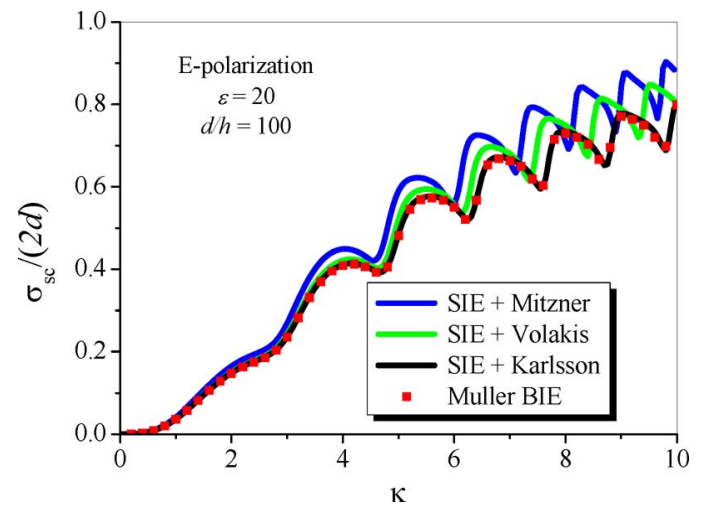

Fig. 4. Normalized RCS versus normalized frequency for a dielectric strip of $\varepsilon=20$ and thickness $h / d=0.01$ under the normal $(\beta=\pi / 2)$ incidence of the E-polarized plane wave.

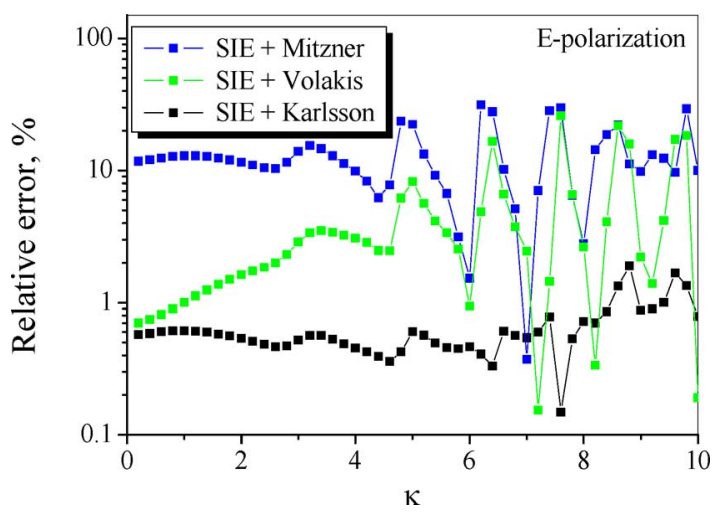

Fig. 5. Relative errors as a function of the normalized frequency for the same configuration as in Fig. 4.

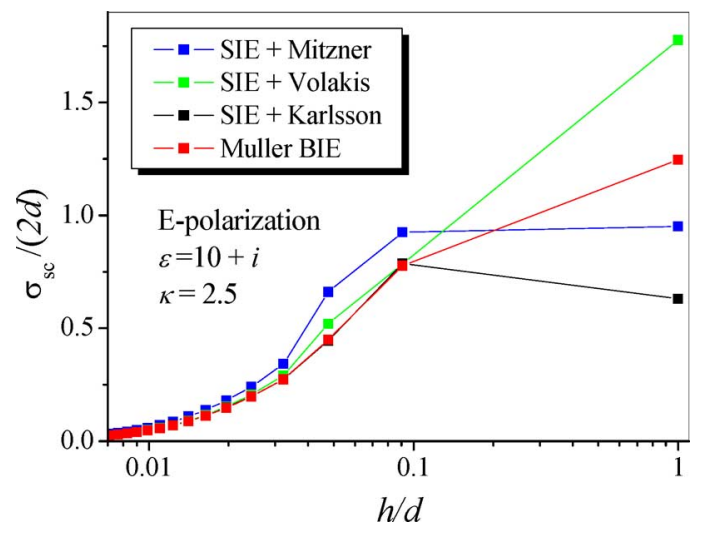

Fig. 6. Normalized TSCS versus $d / h$ for a lossy dielectric strip with of $\varepsilon=$ $10+i$ for $\kappa=2.5$ under the normally incident $(\beta=\pi / 2)$ E-polarized wave.

The plots of TSCS versus thickness for the same strip are given in Fig. 6 and show that the GBC-SIE fails if $h>0.1 d$ (as $k_{0} h>0.5$ ).

\section{B. Dielectric Strip, H-Polarization}

In Fig. 7, we present TSCS versus the normalized frequency for a strip with $\varepsilon=20$ and thickness $h / d=0.01$, under the normally incident $\mathrm{H}$-wave. As one can see, in the H-case the compensated high-contrast model of (14) agrees very well with the MBIE reference data.

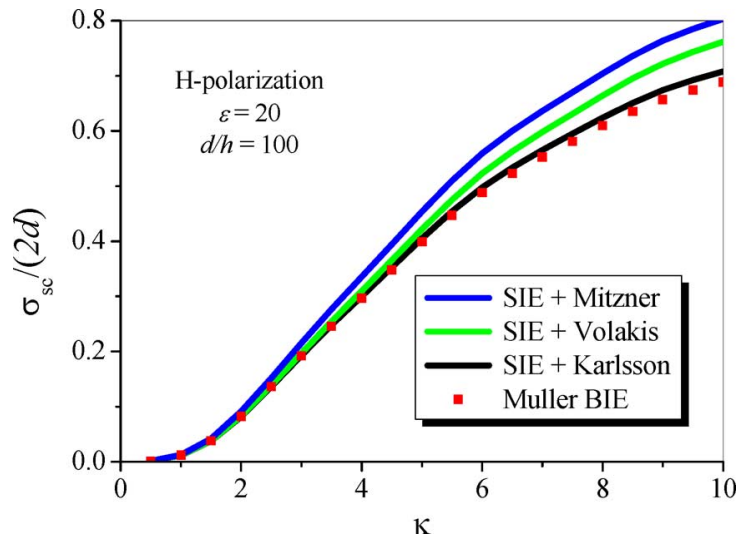

Fig. 7. The same as in Fig. 4 however for the H-polarization case.

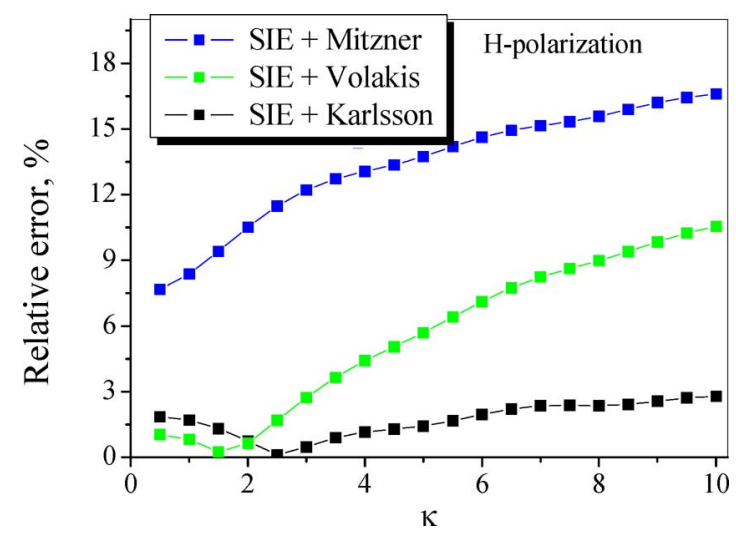

Fig. 8. The same as in Fig. 5 however for the H-polarization case.

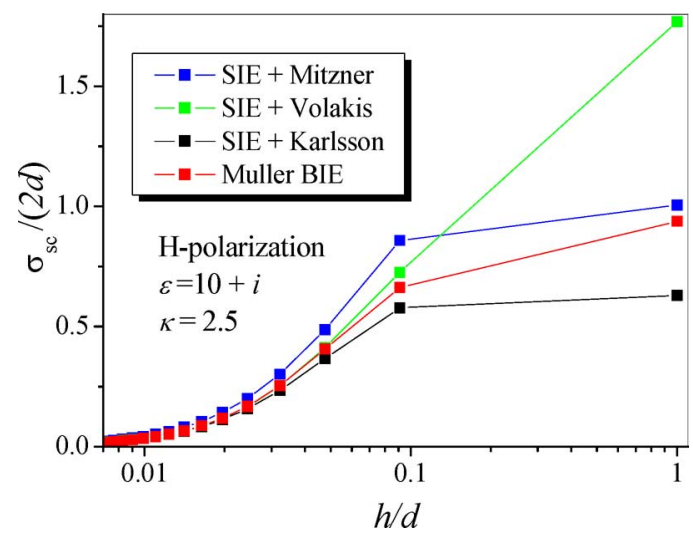

Fig. 9. The same as in Fig. 6 however for the H-polarization case.

The corresponding relative errors are shown in Fig. 8: the models of (14) and (12) provide $2 \%$ and $10 \%$ or better accuracy, respectively.

The plots of TSCS versus the thickness for the same strip in the H-wave case are given in Fig. 9. They demonstrate accuracy of the model (14) within $10 \%$ if only $h<0.1 d$

\section{Metal Strip, E-Polarization}

In the visible range, good metals are "negative dielectrics": for silver, $\operatorname{Re} \varepsilon(\lambda)$ has negative values between -1 and -40 [24]. The plots of TSCS versus the wavelength are shown in Fig. 10 for four different-width silver nanostrips.

As visible, in the E-wave case the scattering of light by thin metal strips can be successfully modeled using the GBC-SIE 


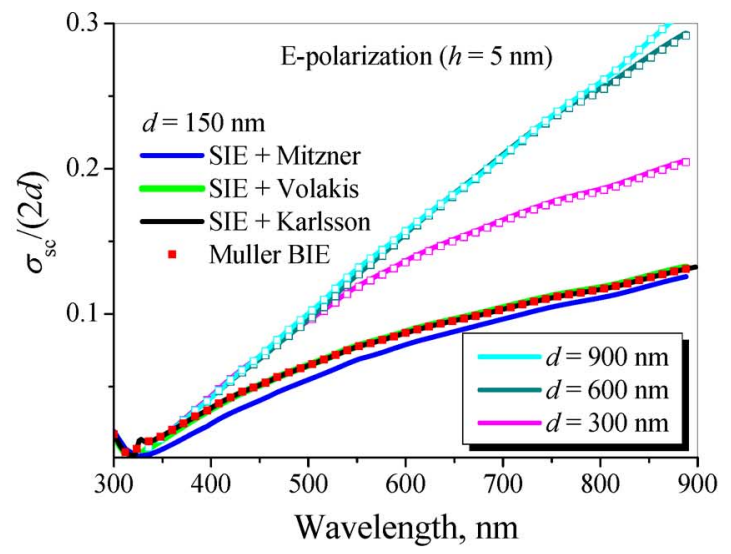

(a)

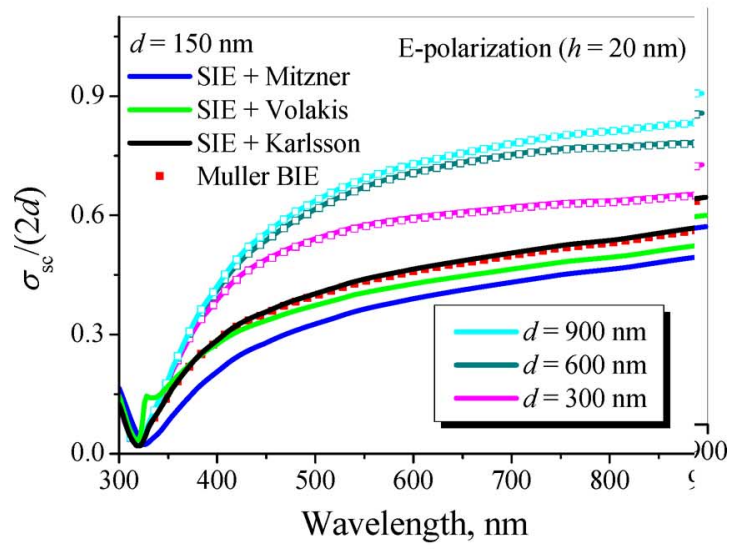

(b)

Fig. 10. Normalized TSCS spectra for the silver strips of $h=5 \mathrm{~mm}$ (a) and $h=20 \mathrm{~mm}$ (b) and varying width, under the normally incident E-polarized wave.

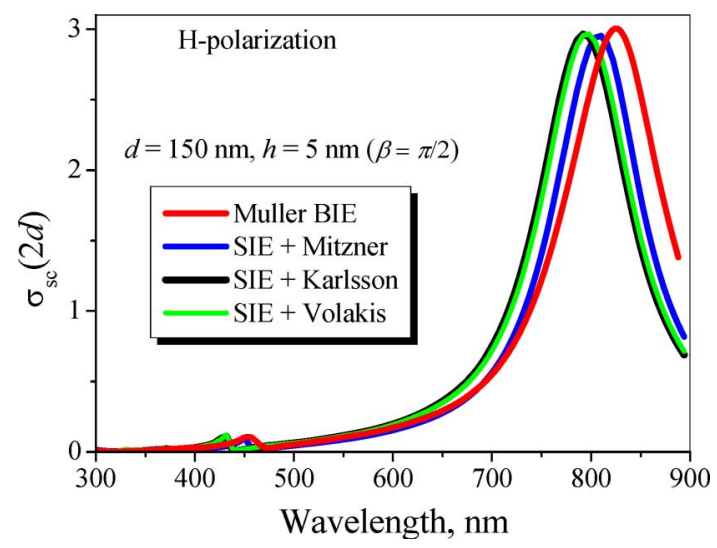

Fig. 11. Normalized TSCS versus the wavelength for the silver strip of $d=$ $150 \mathrm{~mm}$ and $d=5 \mathrm{~mm}$ under the normally incident $\mathrm{H}$-polarized wave.

approach with compensated resistivities (14). For this model, the relative errors in TSCS for two values of thickness, $5 \mathrm{~nm}$ and $20 \mathrm{~nm}$, are about $1 \%$ in the whole visible range. Note also that, in this range, the skin-depth of silver is around $20 \mathrm{~nm}$.

\section{Metal Strip, H-Polarization and Width Correction}

Unlike the E-case, the H-wave scattering by a thin silver nanostrip shows intensive plasmon resonances [4]. Thus the accuracy of an approximate model strongly depends on its ability to place the resonances at correct $\lambda$-see Figs. 11-13.

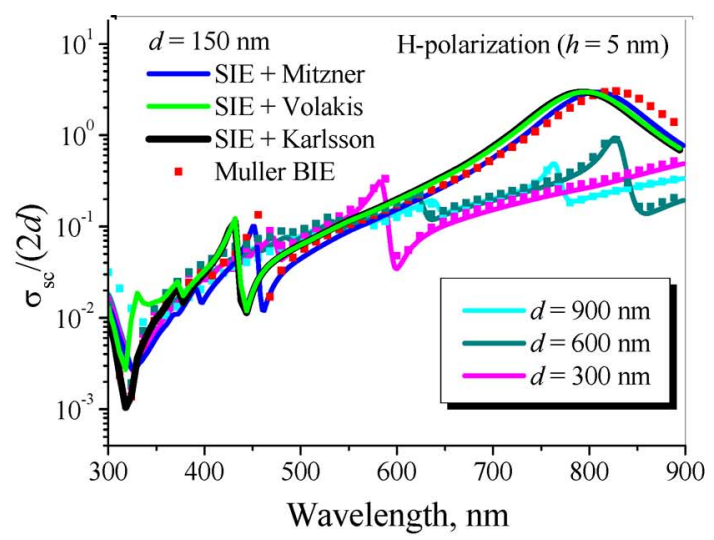

(a)

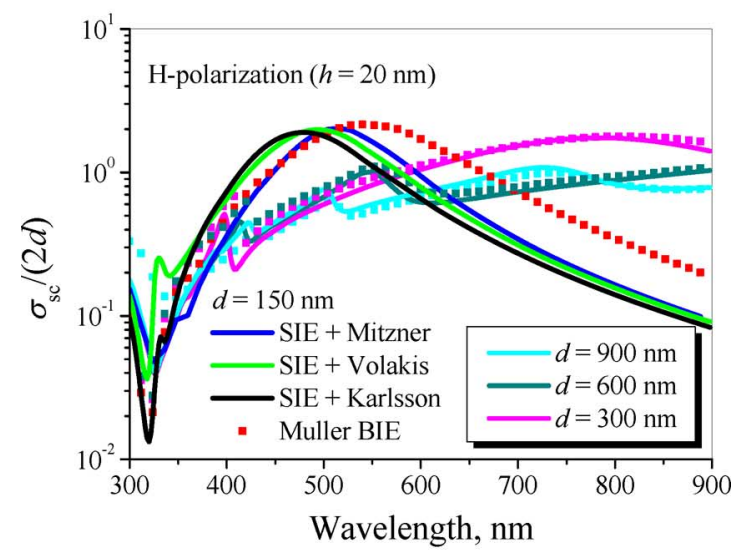

(b)

Fig. 12. The same as in Fig. 10 however for the normally incident $\mathrm{H}$-wave calculated using GBC of (12) (solid curves) and MBIE method (rectangles).

For a 5-nm thin strip [Figs. 11 and 12(a)], all three approximate models reproduce plasmon resonances of TSCS however the shift from correct $\lambda$ is $2 \%$ for (12), $4 \%$ for (14) and $6 \%$ for (13). For a 20-nm thin strip, similar pattern is seen in Fig. 12(b). Thus, the high-contrast model of (12) is relatively the best for the simulation of silver nanostrip scattering. The associated errors of this model with respect to MBIE are shown in Fig. 13 by the lines marked with dots.

They display less than $10 \%$ values except of the vicinities of resonances where they can reach $35 \%$ depending on the thickness. Searching for a remedy, we have found a simple empirical rule allowing us to improve the relative error of the median-line GBC-SIE with resistivities of (12) in 3 to 6 times, in the visible range. Namely, such a nearly best fit is achieved by increasing the width of the strip, in computations, by the value of strip thickness, i.e., to $d_{\text {eff }}=d+h$. Numerical experiments have shown that this empirical correction works well if the strip width is at least between $150 \mathrm{~nm}$ and $450 \mathrm{~nm}$. It results in reducing the relative error of model (12) below 7\% (4\% off resonances) for $h=5 \mathrm{~mm}$ and below $11 \%$ for $h=20 \mathrm{~mm}$, in the whole visible range as demonstrated in Fig. 13 by the curves marked with triangles.

As one can see, wider nanostrips made of silver are generally better simulated with high-contrast model of (12) than narrower ones, although the difference is not significant. 


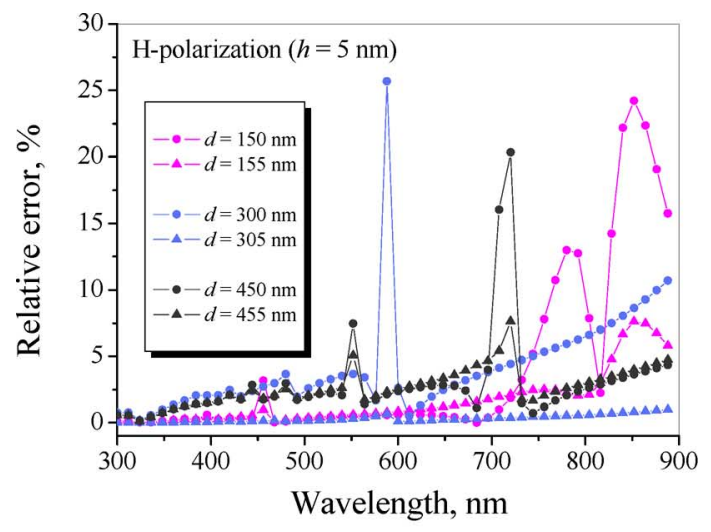

(a)

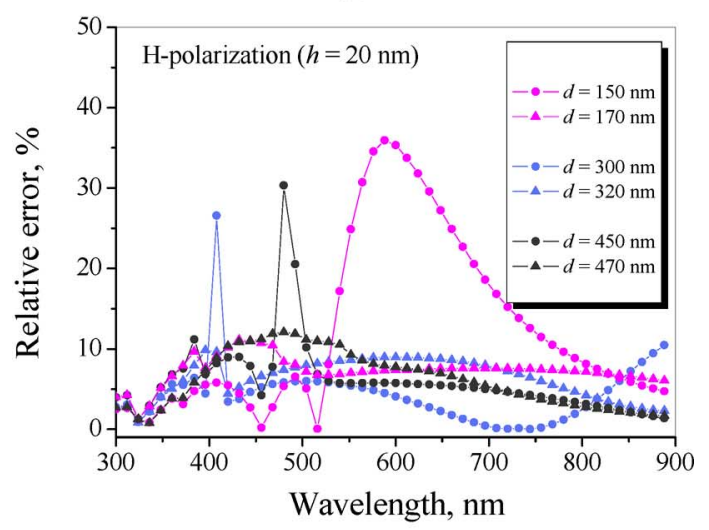

(b)

Fig. 13. Relative error of the TSCS calculation via resistive-strip model from Muller BIE as a function of the wavelength for the different values of the strip width of $h=5 \mathrm{~mm}$ (a) and $20 \mathrm{~nm}$ (b) for different values of $d$ under the normally $(\beta=\pi / 2)$ incident $\mathrm{H}$-polarized plane wave.

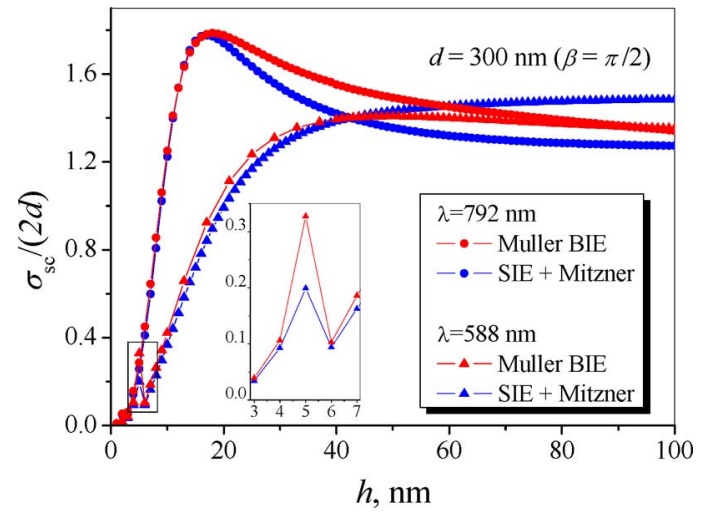

Fig. 14. Normalized TSCS versus the strip thickness $h$ for the silver strip of width $d=300 \mathrm{~mm}$ for fixed wavelengths $\lambda=588 \mathrm{~mm}$ (rectangles) and $\lambda=$ $792 \mathrm{~mm}$ (circles) under the normal incident $\mathrm{H}$-polarized plane wave.

The effect of the metal strip thickness on the accuracy of GBC-SIE can be understood from the results shown in Fig. 14. Generally good coincidence (with error of $2 \%$ to $10 \%$ ), is observed in almost whole range of silver strips thicknesses considered, i.e., up to $100 \mathrm{~nm}$, except of resonance thickness. For example, in Fig. 14 the largest discrepancy is seen at $h=5 \mathrm{~mm}$ for $\lambda=588 \mathrm{~mm}$, which is the resonance wavelength for the strip of this size [compare with Fig. 12(a)].

As GBC (11) has been derived for an infinite plane layer (see Appendix I) where $\beta=0$ is not possible, its applicability to

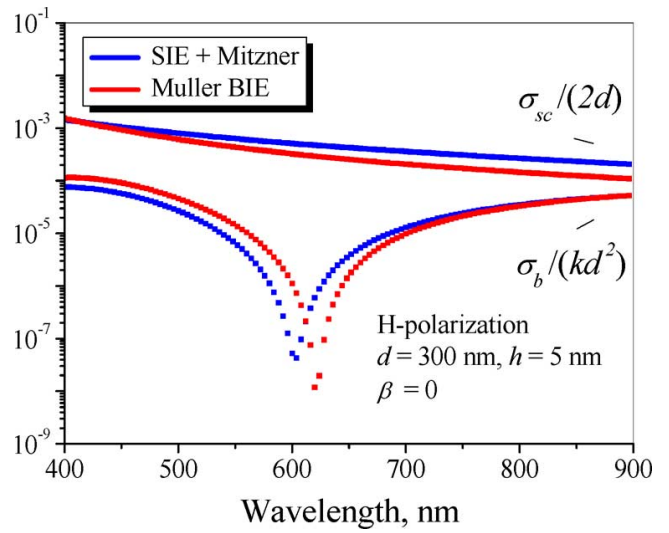

Fig. 15. Normalized TSCS and RCS versus the wavelength for a silver strip of $d=300 \mathrm{~mm}$ and $h=5 \mathrm{~mm}$ under the edge-on $(\beta=0)$ incidence of $\mathrm{H}$-wave.

the case of finite strip scattering can be questioned under the edge-on incidence of a plane wave.

However, as demonstrated by the plots of TSCS and BSCS in Fig. 15, the approximate model of (12) is very accurate even in this case apparently because the surface-plasmon resonances are not excited (they are "dark," i.e., have the symmetry orthogonal to the incident wave). Note that a silver strip under the edge-on incidence is "visible": it both scatters and absorbs the H-polarized light in contrast to the PEC strip, which is invisible. A deep minimum in RCS at $600 \mathrm{~nm}$ is explained by the anti-phase interference of reflections from the leading and trailing edges of the $\lambda / 2$ strip.

\section{CONCLUSION}

We have compared two approaches to the 2-D scattering by thin material strips: approximate model based on the GBC and SIE with a Nystrom-type discretization and accurate model based on the Muller boundary IE discretized with another Nystrom method.

As any empirical technique, GBC-SIE approach has certain limitations. As we have shown, they are mainly associated with the strip thickness and its width: thinner and wider strips show better agreement with reference data obtained from MBIE. Two cases considered, non-dispersive dielectric strip and dispersive metal strip in the visible range demonstrate somewhat different behavior of the errors of approximate technique. Besides, each kind of strips behaves differently for two alternative polarizations.

In the case of conventional-dielectric strip, the E-polarized wave excites resonances (standing waves) associated with the principal surface wave of a corresponding dielectric slab. In the $\mathrm{H}$-case, such resonances are absent. Nevertheless in either polarization, the compensated high-contrast model of (14) is able to provide accuracy of $2 \%$ both in and off resonances.

In the case of metal strip in the visible range, it is the H-polarized wave that excites surface-plasmon resonances absent for the E-polarized light. Here the non-compensated high-contrast model of (12) shows the best performance (error within 10\%) off the resonance. Near the plasmon resonances the same accuracy can be achieved only after changing the metal strip width $d$ to the empirical effective value $d+h$. 


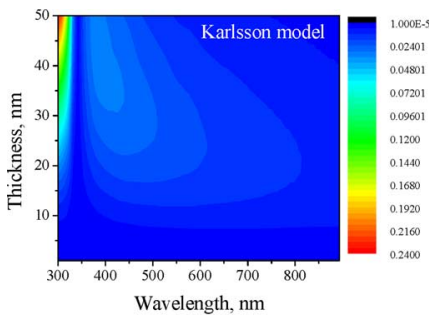

(a)

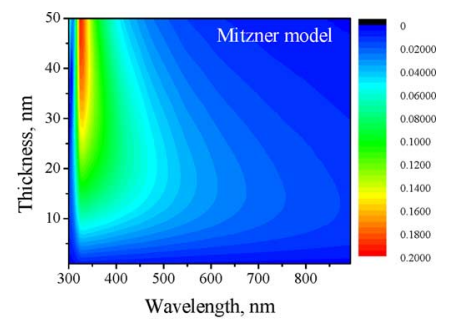

(b)
Fig. 16. Reliefs of the relative error in the reflection coefficient of the $\mathrm{H}$-polarized plane wave computed by Karlsson model (a) and Mitzner model (b), for an infinite silver slab $(\beta=\pi / 4)$, versus the wavelength and the slab thickness .

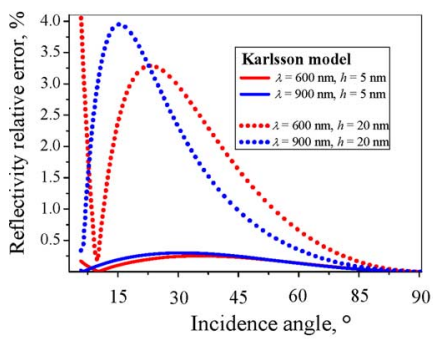

(a)

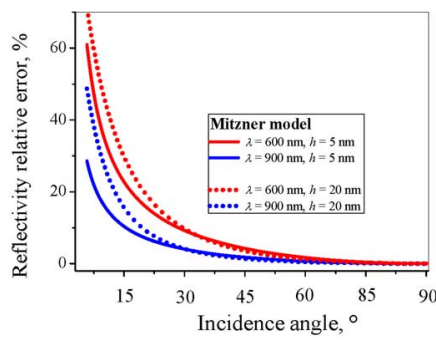

(b)
Fig. 17. The same as in Fig. 16 versus the angle of incidence for a fixed silver slab thickness $h$ and wavelength $\lambda$.

The main advantage of the approximate GBC-SIE technique in comparison to MBIE method is low CPU time cost. The time required for obtaining the TSCS with relative internal accuracy of $10^{-3}$ for a thin dielectric strip or a noble-metal strip in optical range is around $10^{5}$ times larger with MBIE than with GBC-SIE using the optimally selected resistivities.

This advantage makes GBC and associated median-line SIE an effective computational tool to study the scattering and absorption by multiple dielectric and noble-metal strips. In certain cases collecting many dozens and hundreds of tiny scatterers brings new physics. If arranged periodically, they show new resonances caused by the periodicity [4], [25]. These huge collections of scatterers are absolutely out of reach if one uses either in-house MBIE algorithms or commercial software.

\section{APPENDIX I \\ GBC FOR INFINITE SLAB}

The plane-wave scattering by an infinite homogeneous material slab is a classical topic and its analytical solution can be found in many textbooks. The scattering by a zero-thickness sheet characterized with GBC (11) is also solved analytically [3]. Therefore, in Figs. 16 and 17 we show only the error of the GBC in the absolute value of the reflection coefficient of a silver slab in visible range in the $\mathrm{H}$-case.

As one can see, the compensated GBC of Karlsson keeps being very accurate in wider range of thickness values and angles of incidence than non-compensated GBC of Mitzner, although all approximate models fail at the grazing incidence. In the E-polarization scattering by a silver slab, the range of GBC validity is even larger. The same is valid for a conventional-dielectric slab in both polarizations.
TABLE I

COMPUTATIONAL ERror AND TIME VERSUS DiscretizATION ORDER

\begin{tabular}{l|c|c|c|c|c}
\hline \hline \multicolumn{2}{c|}{ GBC-SIE Method with Eqs. (12) } & \multicolumn{3}{|c}{ Muller Boundary IE Method } \\
\hline$N$ & Error, \% & Time, min & $N$ & Error, \% & Time, min \\
\hline 20 & $\underline{\mathbf{0 . 0 5 0 0 4 5 3}}$ & $0 \mathrm{~s} \mathrm{3} \mathrm{ms}$ & 300 & 2.513254 & $1 \mathrm{~min} 7 \mathrm{~s}$ \\
\hline 40 & 0.000494786 & $0 \mathrm{~s} 4 \mathrm{~ms}$ & 450 & 0.605127 & 4 min 6 s \\
\hline 80 & 0.000045572 & 0 s $19 \mathrm{~ms}$ & 600 & 0.270909 & 9 min 6 \\
\hline 160 & 0.000045572 & 0 s $95 \mathrm{~ms}$ & 750 & $\underline{\mathbf{0 . 1 5 4 7 1}}$ & 20 min 4 s \\
\hline 250 & 0.000002472 & $1 \mathrm{~s} 250 \mathrm{~ms}$ & 900 & 0.099723 & 32 min 1 s \\
\hline \hline
\end{tabular}

\section{APPENDIX II \\ CONVERGENCE RATE AND CPU TIME}

To see advantages of the approximate GBC-SIE technique in the context of its convergence and CPU time cost, we have compared computational errors in calculating the TSCS for a silver strip with $d=150 \mathrm{~mm}$ and $h=5 \mathrm{~mm}$ at the fixed wavelength $\lambda=600 \mathrm{~mm}$ in the case of the $\mathrm{H}$-polarization versus the order of discretization $N$ (which is the number of the contour partitions for MBIE and the order of interpolation polynomial for GBC-SIE). These data are collected in Table I.

They demonstrate that a $10^{-3}$ relative internal accuracy in TSCS for a fixed wavelength and fixed silver-strip geometry is provided in $10^{5}$ times faster with GBC-SIE technique than with MBIE one. In both cases we used similar personal computer Intel (R) Core (TM) 2 Quand CPU Q8200@2.33 GHz with RAM-3.25 GB (Windows7 64-bit).

\section{REFERENCES}

[1] O. V. Shapoval, R. Sauleau, and A. I. Nosich, "Scattering and absorption of waves by flat material strips analyzed using generalized boundary conditions and Nystrom-type algorithm," IEEE Trans. Antennas Propag., vol. 59, no. 9, pp. 3339-3346, Sep. 2011.

[2] T. B. A. Senior and J. L. Volakis, "Sheet simulation of a thin dielectric layer," Radio Sci., vol. 22, no. 7, pp. 1261-1272, 1987.

[3] E. Bleszynski, M. Bleszynski, and T. Jaroszewicz, "Surface-integral equations for electromagnetic scattering from impenetrable and penetrable sheets," IEEE Antennas Propag. Mag., vol. 36, no. 6, pp. 14-25, Dec. 1993.

[4] O. V. Shapoval, R. Sauleau, and A. I. Nosich, "Modeling of plasmon resonances of multiple flat noble-metal nanostrips with a median-line integral equation technique," IEEE Trans. Nanotechnol., vol. 12, no. 3, pp. 442-449, May 2013.

[5] A. F. Peterson, "The 'interior resonance' problem associated with surface integral equations of electromagnetics: Numerical consequences and a survey of remedies," Electromagnetics, vol. 10, no. 3, pp. 293-312, 1990

[6] D. Wilton, "Review of current status and trends in the use of integral equations in computational electromagnetics," Electromagnetics, vol. 12, no. 3-4, pp. 287-341, 1992.

[7] S. V. Boriskina, T. M. Benson, P. Sewell, and A. I. Nosich, "Q factor and emission pattern control of the WG modes in notched microdisk resonators," IEEE J. Sel. Topics Quant. Electron., vol. 12, no. 1, pp. 52-58, Jan.-Feb. 2006.

[8] E. I. Smotrova, V. Tsvirkun, I. Gozhyk, C. Lafargue, C. Ulysse, M. Lebental, and A. I. Nosich, "Spectra, thresholds and modal fields of a kite-shaped microcavity laser," J. Opt. Soc. Amer. B, vol. 40, no. 6, pp. 1732-1742, 2013. 
[9] J. L. Tsalamengas, "Exponentially converging Nystrom method in oblique diffraction of arbitrarily polarized waves by bianisotropic/chiral cylinders with arbitrary smooth cross section," IEEE Trans. Antennas Propag., vol. 61, no. 7, pp. 3362-3673, Jul. 2013.

[10] C. Muller, Foundations of the Mathematical Theory of Electromagnetic Waves. Berlin, Germany: Springer, 1969.

[11] N. M. Gunter, Potential Theory and its Applications to Basic Problems of Mathematical Physics. New York, NY, USA: Frederick Ungar, 1967.

[12] S. R. Dubertrand, E. Bogomolny, N. Djellali, M. Lebental, and C. Schmit, "Circular dielectric cavity and its deformations," Phys. Rev. A., vol. 77, no. 1, p. 013804/16, 2008.

[13] K. M. Mitzner, "Effective boundary conditions for reflection and transmission by an absorbing shell of arbitrary shape," IEEE Trans. Antennas Propag., vol. AP-16, no. 6, pp. 706-712, Nov. 1968.

[14] R. F. Harrington and J. R. Mautz, "An impedance sheet approximation for thin dielectric shells," IEEE Trans. Antennas Propag., vol. AP-36, no. 4, pp. 531-534, Jul. 1975.

[15] J. L. Volakis, "High-frequency scattering by a thin material half-plane and strip," Radio Science, vol. 23, no. 3, pp. 450-462, 1988.

[16] G. Bouchitté, "Analyse limite de la diffraction d'ondes électromagnétiques par une structure mince," C. R. Acad. Paris, ser. II, vol. 311, pp. $51-56,1990$.

[17] A. Karlsson, "Approximate boundary conditions for thin structures," IEEE Trans. Antennas Propag., vol. 57, no. 1, pp. 144-148, Jan. 2009.

[18] Z. Nazarchuk and K. Kobayashi, "Mathematical modelling of electromagnetic scattering from a thin penetrable target," Progress Electromag. Res., vol. 55, pp. 95-116, 2005.

[19] I. M. Braver, P. S. Fridberg, K. L. Garb, and I. M. Yakover, "The behavior of the electromagnetic field near the edge of a resistive halfplane," IEEE Trans. Antennas Propag., vol. 36, no. 12, pp. 1760-1768, Dec. 1988.

[20] T. L. Zinenko, A. I. Nosich, and Y. Okuno, "Plane wave scattering and absorption by resistive-strip and dielectric-strip periodic gratings," IEEE Trans. Antennas Propag., vol. 46, no. 10, pp. 1498-1505, Oct. 1998.

[21] T. L. Zinenko, M. Marciniak, and A. I. Nosich, "Accurate analysis of light scattering and absorption by an infinite flat grating of thin silver nanostrips in free space using the method of analytical regularization," IEEE J. Sel. Top. Quant. Electron., vol. 19, no. 3, p. 9000108/8, MayJun. 2013.

[22] J. Tsalamengas, “Exponentially converging Nystrom's methods for systems of SIEs with applications to open/closed strip or slot-loaded 2-D structures," IEEE Trans. Antennas Propag., vol. 54, no. 5, pp. 1549-1558, May 2006.

[23] J. H. Richmond, "Scattering by a thin dielectric strip," IEEE Trans. Antennas Propag., vol. AP-33, no. 1, pp. 64-68, Jan. 1985.

[24] R. W. Christy and P. B. Johnson, "Optical constants of the noble metals," Phys. Rev. B, vol. 6, no. 12, pp. 4370-4379, 1972.

[25] O. V. Shapoval and A. I. Nosich, "Finite gratings of many thin silver nanostrips: Optical resonances and role of periodicity," AIP Advances, vol. 3, no. 4, p. 042120/13, 2013.

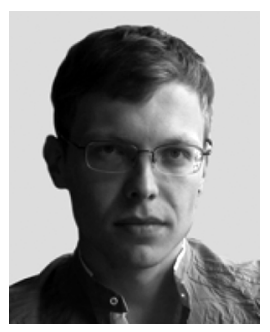

Ilya O. Sukharevsky ( $\mathrm{S}^{\prime} 08-\mathrm{M}^{\prime} 13$ ) was born in Kharkiv, Ukraine, in 1984. He received the B.Sc. and M.Sc. degrees in mathematics from the Kharkiv National University, Kharkiv, Ukraine, and the $\mathrm{Ph} . \mathrm{D}$. degree from the Institute of Radio-Physics and Electronics, National Academy of Sciences of Ukraine, Kharkiv, Ukraine, in 2005, 2006, and 2011 respectively.

Since 2013, he has been a Post-Doctoral Researcher at the Communication and Spectrum Management Research Center (ISYAM), Bilkent University, Ankara, Turkey. His scientific interests are in the integral equation techniques and asymptotic methods of electromagnetics.

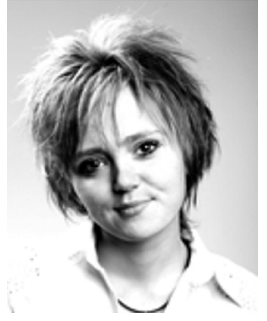

Olga V. Shapoval (S'10) was born in Nikopol, Ukraine, in 1987. She received the M.S. degree (with honors) in applied mathematics from Kharkiv National University, Kharkiv, Ukraine, in 2009. She is currently working toward the Ph.D. degree in radio physics at the Laboratory of Micro and Nano-Optics, Institute of Radio-Physics and Electronics, National Academy of Science of Ukraine, Kharkiv.

Her current research interests are in the scattering problems by striplike structures, efficient mathematical and numerical solution techniques, and singular and hyper-singular integral equations.

Ms. Shapoval was a recipient in 2010 of the URSI Young Scientist Award for attending the Asia-Pacific Radio Science Conference in Toyama and the Doctoral Research Award of the IEEE Antennas and Propagation Society. In 2013, she received the URSI Young Scientist Award for attending the Electromagnetic Theory Symposium in Hiroshima. In 2014, she was a recipient of the TICRA Foundation Award for Young Scientists.

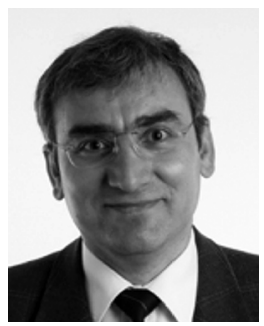

Ayhan Altintas (SM'93) received the B.S. and M.S. degrees from the Middle East Technical University, Ankara, Turkey, and the Ph.D. degree from Ohio State University, Columbus, OH, USA, in 1979,1981 , and 1986, respectively, all in electrical engineering.

Currently, he is Professor of Electrical Engineering at Bilkent University, Ankara, Turkey. He is also the Director of Communication and Spectrum Management Research Center (ISYAM).

Prof. Altintas was Chairman of the IEEE Turkey Section in 1991-1993 and 1995-1997. He is the founder and first chair of the IEEE AP/MTT Chapter in the Turkey Section. At present, he is the President of URSI Turkish National Committee. His research interests are in electromagnetics, antennas, propagation, and wireless communication systems. He is a Fulbright Scholar, and an Alexander von Humboldt Fellow. He received the Ohio State University ElectroScience Laboratory Outstanding Dissertation Award of 1986, IEEE 1991 Outstanding Student Branch Counselor Award, 1991 Research Award of Prof. M. N. Parlar foundation of METU, and Young Scientist Award of Scientific and Technical Research Council of Turkey in 1996. He is a member of Sigma Xi and Phi Kappa Phi and a recipient of IEEE Third Millennium Medal.

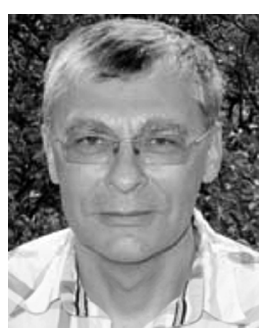

Alexander I. Nosich (M'94-SM'95-F'04) was born in Kharkiv, Ukraine, in 1953. He received the M.S., Ph.D., and D.Sc. degrees in radio physics from the Kharkiv National University, Kharkiv, Ukraine, in 1975,1979 , and 1990, respectively.

Since 1979, he has been with the Institute of Radio Physics and Electronics, National Academy of Science of Ukraine, Kharkiv, where he is currently Professor and Principal Scientist heading the Laboratory of Micro and Nano Optics. Since 1992, he has held a number of guest fellowship and professorship in the EU, Japan, Singapore, and Turkey. His research interests include the method of analytical regularization, propagation and scattering of waves in open waveguides, simulation of the semiconductor lasers and antennas, and the history of microwaves.

Prof. Nosich was one of the initiators and technical committee chairman of the international conference series on Mathematical Methods in Electromagnetic Theory (MMET). In 1995, he organized IEEE AP-S East Ukraine Chapter, the first one in the former USSR. Currently he represents Ukraine in the European Association on Antennas and Propagation. 